Pacific Journal of Mathematics

STABLE ISOMORPHISM OF HEREDITARY SUBALGEBRAS OF 


\title{
STABLE ISOMORPHISM OF HEREDITARY SUBALGEBRAS OF $C^{*}$-ALGEBRAS
}

\author{
LAWRENCE G. BROWN
}

The main theorem is that if $B$ is a hereditary $C^{*}$-subalgebra of $A$ which is not contained in any proper closed two-sided ideal, then under a suitable separability hypothesis $A \otimes \mathscr{K}$ is isomorphic to $B \otimes \mathscr{K}$, where $\mathscr{K}$ is the $C^{*}$-algebra of compact operators on a separable infinite-dimensional Hilbert space. In the special case where $A=C \otimes \mathscr{K}$ and $B=p A p$ for some projection $p$ in the double centralizer algebra, $M(A)$, of $A$ such that $p$ commutes with $C \otimes 1 \subset M(A)$, the theorem follows from a result of Dixmier and Douady [12]. In fact $p$ must be defined by a continuous mapping from the spectrum $\hat{C}$ of $C$ to the strong grassmanian. Thus $p$ defines a continuous field of Hlibert spaces on $\hat{C}$ and [12] implies that the countable direct sum of this field with itself is trivial. Our proof amounts to an abstraction of [12]. The theorem also leads to an abstraction and generalization of some results of Douglas, Fillmore, and us on extensions of $C^{*}$-algebras $([6, \S 3])$. The final section of the paper contains a generalization of the Dauns-Hofmann theorem which is needed to justify some of our remarks.

1. Preliminaries. If $B$ is a $C^{*}$-subalgebra of $A, B$ is called hereditary if $a \in A, b \in B, 0 \leqq a \leqq b$ imply $a \in B$. $B$ is full if Iit is not contained in any proper closed two-sided ideal of $A$.

We will be particularly concerned with hereditary subalgebras which are related to the double centralizer algebra, $M(A)$, of $A$. An element of $M(A)$ is a pair $x=(S, T)$, where $S$ and $T$ are linear operators on $A$ such that $a \cdot S(b)=T(a) \cdot b$ for all $a, b \in A . M(A)$ is the universal $C^{*}$-algebra containing $A$ as a two-sided ideal. $S$ and $T$ are the restrictions to $A$ of the left and right multiplications by $x$. The strict topology of $M(A)$ is the weakest topology in which the maps $x \rightarrow x \alpha$ and $a \rightarrow a x$ are continuous for each $a \in A$, where $A$ has the norm topology. Any nondegenerate representation, $\pi$, of $A$ extends uniquely to a representation, $\tilde{\pi}$, of $M(A)$ on the same Hilbert space. For further details on $M(A)$ the reader is referred to [8] and [3].

If $p$ is a projection in $M(A), p A p$ is a hereditary subalgebra of $A$ which will be called a corner.

LEMma 1.1. If $p$ is a projection in $M(A)$, the following are equivalent:

1. For any nondegenerate representation, $\pi$, of $A, \tilde{\pi}(p) \neq 0$. 
2. For any irreducible representation, $\pi$, of $A, \tilde{\pi}(p) \neq 0$.

3. $p A p$ is full.

4. $p A$ is not contained in any proper closed two-sided ideal of A.

5. Ap is not contained in any proper closed two-sided ideal of A.

6. The norm-closed two-sided ideal of $M(A)$ generated by $p$ includes $A$.

7. $p$ is not contained in any proper strictly closed two-sided ideal of $M(A)$.

Proof. $1 \Rightarrow 2$ is obvious.

$2 \Rightarrow 3$. If $p A p$ is not full, there must be an irreducible representation $\pi$ which vanishes on the two-sided ideal generated by $p A p$. Thus $\tilde{\pi}(p) \cdot \pi(A) \cdot \tilde{\pi}(p)=0$. Since the strong closure of $\pi(A)$ contains $1, \tilde{\pi}(p)=0$.

$3 \Rightarrow 4$ and 5. Obvious because $p A p=p A \cap A p$.

4 or $5 \Rightarrow 6$. The ideal considered in 6 obviously includes $p A$ and Ap.

$6 \Rightarrow 7 . \quad A$ is strictly dense in $M(A)$.

$7 \Rightarrow 1$. The kernel of $\tilde{\pi}$ is strictly closed. Thus if $\tilde{\pi}(p)=0$ and $p$ generates a strictly dense ideal, $\tilde{\pi}$ and $\pi$ must be 0 .

$p$ will be called full if it satisfies the conditions of 1.1 .

A hereditary subalgebra need not be a corner. Let $A^{* *}$ be the Banach space double dual of $A . A^{* *}$ is a $W^{*}$-algebra, and any nondegenerate representation, $\pi$, of $A$ extends uniquely to a $W^{*}$-representation, $\pi^{* *}$, of $A^{* *}$. The hereditary subalgebras of $A$ correspond one-to-one to the open projections (see [2]) of $A^{* *}$. The subalgebra corresponding to $p$ is $\left(p A^{* *} p\right) \cap A$, and $p$ is open if and only if this is weak* dense in $p A^{* *} p$.

Lemma 1.2. If $B$ is the hereditary subalgebra of $A$ corresponding to the open projection $p$, the following are equivalent:

1. For any nondegenerate representation, $\pi$, of $A, \pi^{* *}(p) \neq 0$.

2. For any irreducible representation, $\pi$, of $A, \pi^{* *}(p) \neq 0$.

3. $B$ is full.

4. The norm-closed two-sided ideal of $A^{* *}$ generated by $p$ includes $A$.

5. $p$ is not contained in any proper weak*-closed two-sided $i d e a l$ of $A^{* *}$.

Proof. $1 \Rightarrow 2$ is obvious.

$2 \Rightarrow 3$. If $B$ is not full, some irreducible representation, $\pi$, 
vanishes on $B$. Since $p$ is in the weak ${ }^{*}$ closure of $B$ and $\pi^{* *}$ is continuous from the weak* topology of $A^{* *}$ to the weak operator topology, $\pi^{* *}(p)=0$.

$3=4$. The ideal of 4 includes $B$.

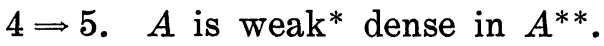

$5 \Rightarrow 1$. If $\pi^{* *}(p)=0,5$ and the weak ${ }^{*}$ continuity of $\pi^{* *}$ imply $\pi^{* *}$ and $\pi=0$.

If $A$ and $B$ are $C^{*}$-algebras $A \otimes_{\min } B$ denotes the completion of the algebraic tensor product with respect to the smallest $C^{*}$-norm. If $\pi$ and $\rho$ are faithful representations of $A$ and $B, \pi \otimes \rho$ is a faithful representation of $A \otimes_{\min } B$. The abbreviated symbol $A \otimes B$ will be used if all $C^{*}$ tensor products are known to agree. See [15] and its references for further details on tensor products. $\mathscr{L}(\mathscr{H})$ denotes the algebra of bounded operators on the Hilbert space $\mathscr{H}$ and $\mathscr{K}(\mathscr{H})$ the ideal of compact operators. The abbreviated symbols $\mathscr{L}$ and $\mathscr{K}$ may be used if $\mathscr{H}$ is separable and infinitedimensional. $C^{*}$-algebras $A$ and $B$ are called stably isomorphic if $A \otimes \mathscr{K}$ and $B \otimes \mathscr{K}$ are isomorphic. $A$ is stable if $A$ and $A \otimes \mathscr{K}$ are isomorphic. A positive element $e$ of a $C^{*}$-algebra $A$ is called strictly positive if $\phi(e)>0$ for every state $\phi$ of $A$. Two equivalent conditions are that $A e$ be norm dense in $A$ and that $e$ not be contained in any proper hereditary $C^{*}$-subalgebra of $A$. $A$ has a strictly positive element if and only if $A$ has a countable approximate identity, and this is always so if $A$ is separable. (See [1].) $\hat{A}$, the spectrum of $A$, denotes the set of equivalence classes of irreducible representations of $A$ with the hull-kernel topology. ([10]).

A continuous field of Hilbert spaces $E$, on a topological space $X$ is given by $\left(\left\{E_{x}\right\}, \Gamma\right)$, where $E_{x}$ is a Hilbert space for each $x \in X$ and $\Gamma$ is a space of functions $\phi$ on $X$ such that $\phi(x) \in E_{x}$ and certain axioms are satisfied. See [12] for the precise definition and further details. The strong grassmanian, $\mathscr{G}$, is the set of projections in $\mathscr{L}$ with the strong operator topology. A continuous function, $f: X \rightarrow \mathscr{G}$, give rise to a continuous field which is a complimented subfield of the countable rank trivial field. $f_{1}$ and $f_{2}$ give rise to isomorphic fields if and only if there is a (double-) strongly continuous function $u: X \rightarrow \mathscr{L}$ such that $u^{*}(x) \cdot u(x)=f_{1}(x)$ and $u(x) \cdot u^{*}(x)=f_{2}(x)$ for all $x \in X$.

If $A$ is a separable $C^{*}$-algebra, $\operatorname{Ext}(A)$ is the set of equivalence classes of ${ }^{*}$-monomorphisms $\tau: A \rightarrow \mathscr{L} / \mathscr{K}$. $\operatorname{Ext}(A)$ has a natural semigroup structure and is known to be a group in many cases. $A$ *-monomorphism $\theta: A \rightarrow B$ induces a homomorphism $\theta^{*}: \operatorname{Ext}(B) \rightarrow$ $\operatorname{Ext}(A)$ by $\tau \rightarrow \tau \circ \theta \cdot{ }^{1} \quad \operatorname{Ext}(A) \cong \operatorname{Ext}(A \otimes \mathscr{K})$ and hence $\operatorname{Ext}(A) \cong$ $\operatorname{Ext}(B)$ whenever $A$ and $B$ are stably isomorphic. For further details see [6], [5], [17], [9]. 
2. The main theorem. If $I$ is a dense two-sided ideal of the $C^{*}$-algebra $A, 1.7 .2$ of [10] asserts the existence of an increasing approximate identity of $A$ consisting of elements of $I$. It is convenient to begin with a slight refinement of this.

THEOREM 2.1. If $R$ is a (not necessarily closed) right ideal of $A$ which generates a dense two-sided ideal, then $A$ has an increasing approximate identity consisting of finite sums $\sum r_{j}^{*} r_{j}, r_{j} \in R$.

Proof. We apply the argments of [10] to the dense two-sided ideal $I=R^{*} \cdot R$. Only one new point arises: We need to know that for any self-adjoint element, $x$, of $I, x \leqq \sum c_{j}^{*} c_{j}$ for suitable $c_{j} \in R$. This follows from $2 \cdot \operatorname{Re} a^{*} b=a^{*} b+b^{*} a \leqq a^{*} a+b^{*} b$. A typical member of the approximate identity will be given by $r_{j}=c_{j}\left(\varepsilon+\sum c_{k}^{*} c_{k}\right)^{-1 / 2}$, for $\varepsilon>0$.

Lemma 2.2. If $p$ is a full projection in $M(A), e \in A$, and $\varepsilon>0$, then there are $a_{1}, a_{2}, \cdots, a_{n} \in A$ such that $\sum a_{i}^{*} p a_{i} \leqq 1$ and

$$
\left\|\left(1-\sum a_{i}^{*} p a_{i}\right) e\right\|<\varepsilon .
$$

Proof. Apply 2.1 to $R=p A$. Choose the $a_{i}$ so that $\sum a_{i}^{*} p a_{i}$ is a suitable member of the approximate identity.

LEMMA 2.3. If $p$ is a full projection in $M(A)$ and $A$ has a strictly positive element, $e$, then there are $a_{1}, a_{2}, \cdots, \in A$ such that $\sum_{1}^{\infty} a_{i}^{*} p a_{i}=1$, with convergence in the strict topology of $M(A)$.

Proof. We construct recursively $n_{1}<n_{2}<\cdots n_{k}$ and $a_{i}, 1 \leqq i \leqq n_{k}$, such that with $s_{k}=\sum_{1}^{n_{k}} a_{i}^{*} p a_{i}, s_{k} \leqq 1$ and $\left\|\left(1-s_{k}\right) e\right\| \leqq 1 / k$. Suppose this is given for $k$. By 2.2 we choose $b_{1}, \cdots, b_{m} \in A$ such that with $s^{\prime}=\sum b_{j}^{*} p b_{j}, s^{\prime} \leqq 1$ and $\left\|\left(1-s_{k}\right)^{1 / 2}\left(1-s^{\prime}\right)\left(1-s_{k}\right)^{1 / 2} e\right\|<1 / k+1$. Let $n_{k+1}=n_{k}+m$ and $a_{n_{k}+j}=b_{j}\left(1-s_{k}\right)^{1 / 2}$.

Since $e \cdot A$ is dense in $A$, it is now clear that $s_{k}$ converges strictly to 1 . For $x \in A$ the sequence $x^{*}\left(1-\sum_{1}^{n} \alpha_{i}^{*} p a_{i}\right) x$ is monotone decreasing and a subsequence converges to 0 . Hence the sequence converges to 0 . Since $\left\|\left(1-\sum_{1}^{n} a_{i}^{*} p a_{i}\right) x\right\| \leqq\left\|x^{*}\left(1-\sum_{1}^{n} a_{i}^{*} p a_{i}\right) x\right\|^{1 / 2}$, the lemma is proved.

We fix a set of matrix units $\left\{e_{i j}\right\}$ which generate $\mathscr{K}$. We will consistently use without reference the fact that $M(A) \bigotimes_{\min } M(B)$ is a subalgebra of $M\left(A \otimes_{\min } B\right)$ (cf. [3, 3.8]).

LEMMA 2.4. If $p$ is a full projection in $M(A)$ and $A$ has a

${ }^{1}$ A result of D. Voiculescu, [17], which we do not need here, eliminates the need for $\theta$ to be assumed one-to-one. 
strictly positive element, then there is a partial isometry $u \in$ $M(A \otimes \mathscr{K})$ such that $u^{*} u=1 \otimes e_{11}$ and $u u^{*} \leqq p \otimes 1$.

Proof. Choose $a_{1}, \alpha_{2}, \cdots$ as in 2.3 and let $u=\sum_{1}^{\infty} p \alpha_{i} \otimes e_{i 1}$. It is routine to show that the infinite series converges in the strict topology of $M(A \otimes \mathscr{K})$. In fact, since finite sums $\sum b_{j k} \otimes e_{j k}$ are dense in $A \otimes \mathscr{K}$ and since with $u_{n}=\sum_{1}^{n} p a_{i} \otimes e_{i 1},\left\|u_{n}\right\| \leqq 1\left(u_{n}^{*} u_{n}=\right.$ $\left.\sum_{1}^{n} a_{i}^{*} p a_{i} \otimes e_{11}\right)$, it is sufficient to show that $u_{n}\left(b \otimes e_{j k}\right)$ and $\left(b \otimes e_{j k}\right) u_{n}$ converge for all $b \in A$ and all $j, k$. The second is obvious since $\left(b \otimes e_{j k}\right) \cdot\left(p a_{i} \otimes e_{i 1}\right)=0$ for $i \neq k$. For the first, note that

$$
\begin{aligned}
& \left\|\left(u_{m}-u_{n}\right)\left(b \otimes e_{j k}\right)\right\|^{2} \\
& \quad=\|\left(b^{*} \otimes e_{k j}\right)\left(u_{m}-u_{n}\right)^{*}\left(u_{m}-u_{n}\right)\left(b \otimes e_{j k}\right) \\
& \quad \leqq\left\|b^{*}\left(\sum_{n+1}^{m} a_{i}^{*} p a_{i}\right) b\right\| \longrightarrow 0
\end{aligned}
$$

by 2.3 .

LEMмA 2.5. With the same hypotheses there is $v \in M(A \otimes \mathscr{K})$ such that $v^{*} v=1$ and $v v^{*}=p \otimes 1$.

Proof. Let $N$ be the set of natural numbers and write $N=$ $\bigcup_{1}^{\infty} N_{j}$ where the $N_{j}$ 's are disjoint infinite subsets. Let $e_{j}=\sum_{i \in N_{j}} 1 \otimes$ $e_{i i} \in M(A \otimes \mathscr{K})$. We construct recursively a sequence $\left\{v_{k}\right\}$ of partial isometries in $M(A \otimes \mathscr{K})$ such that $v_{k}^{*} v_{k}$ are mutually orthogonal, $v_{k} v_{k}^{*}$ are mutually orthogonal, $\sum_{1}^{2 n-1} v_{k}^{*} v_{k}=\sum_{1}^{n} e_{j}, \sum_{1}^{2 n} v_{k}^{*} v_{k} \leqq \sum_{1}^{n+1} e_{j}$, $\sum_{1}^{2 n-1} v_{k} v_{k}^{*} \leqq(p \otimes 1) \cdot \sum_{1}^{n} e_{j}$, and $\sum_{1}^{2 n} v_{k} v_{k}^{*}=(p \otimes 1)$. $\sum_{1}^{n} e_{j}$. Once this is done, it is routine to define $v=\sum_{1}^{\infty} v_{k}$ with strict convergence.

To construct $v_{2 n-1}$ we use a partial isometry $w$ such that $w^{*} w=e_{n}$ and $w w^{*} \leqq(p \otimes 1) \cdot e_{n}$. $w$ exists by an easy argument based on 2.4. Let $v_{2 n-1}=w\left(\sum_{1}^{n} e_{j}-\sum_{1}^{2 n-2} v_{k}^{*} v_{k}\right)$. To construct $v_{2 n}$, we use a partial isometry $w^{\prime}$ such that $w^{\prime *} w^{\prime} \leqq e_{n+1}$ and $w^{\prime} w^{\prime *}=(p \otimes 1) \cdot e_{n}$. The existence of $w^{\prime}$ is obvious. Let $v_{2 n}=(p \otimes 1) \cdot\left[\sum_{1}^{n} e_{j}-\sum_{1}^{2 n-1} v_{k} v_{k}^{*}\right] \cdot w^{\prime}$.

CoRollary 2.6. If $A$ has a strictly positive element and $B$ is a full corner of $A$, then $A$ and $B$ are stably isomorphic. The stable isomorphism is induced by a partial isometry in $M(A \otimes \mathscr{K})$.

Proof. $B \otimes \mathscr{K}$ can be identified with $(p \otimes 1) \cdot A \otimes \mathscr{K} \cdot(p \otimes 1)$. The isomorphism of $B \otimes \mathscr{K}$ with $A \otimes \mathscr{K}$ is induced by the $v$ of 2.5 .

CoRollary 2.7. If $A$ is separable and $B$ is a full corner of $A$, then the inclusion $i: B \rightarrow A$ induces an isomorphism $i^{*}: \operatorname{Ext}(A) \rightarrow$ $\operatorname{Ext}(B)$. 
Proof. Consider the commutative diagram:

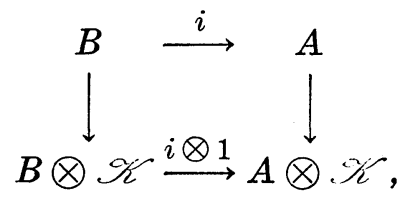

where the vertical maps are given by $a \rightarrow a \otimes e_{11}$. Since the vertical maps induce isomorphisms on Ext by [6,3.15], it is sufficient to show $(i \otimes 1)$ is an isomorphism. But by $[6,3.11] i \otimes 1$ induces the same map from $\operatorname{Ext}(A \otimes \mathscr{K})$ to $\operatorname{Ext}(B \otimes \mathscr{K})$ as the isomorphism of $B \otimes \mathscr{K}$ with $A \otimes . \mathscr{K}$ constructed in 2.6.

Theorem 2.8. If $B$ is a full hereditary $C^{*}$-subalgebra of $A$ and if each of $A$ and $B$ has a strictly positive element, then $B$ is stably isomorphic to $A$. There is a canonical set of isomorphisms from $B \otimes \mathscr{K}$ to $A \otimes \mathscr{K}$ which agree up to automorphisms of $A \otimes \mathscr{K}$ induced by unitary elements of $M(A \otimes \mathscr{K}) .^{2}$

Proof. Let $C$ be the $C^{*}$-subalgebra of $A \otimes M_{2}$ consisting of sums $\sum a_{i j} \otimes e_{i j}$ such that $a_{11} \in B, a_{12} \in \overline{B \cdot A}, a_{21} \in \overline{A \cdot B}$, and $a_{22} \in A$. Here, $M_{2}$ is the algebra of $2 \times 2$ complex matrices and $\left\{e_{i j}: i, j=1,2\right\}$ is a system of matrix units. $B$ is isomorphic to the full corner $B \otimes e_{11}$ of $C$, and $A$ is isomorphic to the full corner $A \otimes e_{22}$. Moreover if $f_{1}$ and $f_{2}$ are strictly positive elements of $B$ and $A$, then $f_{1} \otimes e_{11}+f_{2} \otimes e_{22}$ is a strictly positive element of $C$. Thus 2.6 implies that $A$ and $B$ are stably isomorphic.

The isomorphism from $B \otimes \mathscr{K}$ to $A \otimes \mathscr{K}$ is induced by a partial isometry $v \in M(C \otimes \mathscr{K}) . \quad v$ can be replaced only by $u v$ where $u$ is a partial isometry whose initial and final projections are $1 \otimes e_{22} \otimes$ $1 \in M(C \otimes \mathscr{K})$. Since $M(A \otimes \mathscr{K})$ is a corner of $M(C \otimes \mathscr{K})$ (cf. [3, 4.5]), we may regard $u$ as a unitary in $M(A \otimes \mathscr{C})$. Thus the second sentence of the theorem is proved. (Note that there are no arbitrary choices in the construction of $C$.)

COROLLARy 2.9. If $A$ and $B$ are $C^{*}$-algebras with strictly positive elements, then the following are equivalent:

1. $A$ and $B$ are stably isomorphic.

2. There is a $C^{*}$-algebra $C$ with a strictly positive element such that each of $A$ and $B$ is isomorphic to a full corner of $C$.

3. There is a $C^{*}$-algebra $C$ such that each of $A$ and $B$ is isomorphic to a full hereditary subalgebra of $C$.

${ }^{2}$ The "meaning" of the second sentence is clarified in [7]. 
Proof. $1 \Rightarrow 2$. Take $C=A \otimes \mathscr{K} . A$ is isomorphic to the full corner $A \otimes e_{11}$ of $C . \quad B$ is isomorphic to the full corner $B \otimes e_{11}$ of $B \otimes \mathscr{K}$, which is isomorphic to $C$.

$2 \Rightarrow 3$ is obvious.

$3 \Rightarrow 1$. Since $A$ has a strictly positive element, $A$ contains a separable subalgebra $A_{0}$ such that $A_{0} A A_{0}$ is dense in $A$. Similarly, $B \supset B_{0}$ such that $B_{0} B B_{0}$ is dense in $B$. Let $C_{0}$ be the $C^{*}$-subalgebra of $C$ generated by $A_{0}$ and $B_{0}$ and $C_{1}$ the hereditary $C^{*}$-subalgebra generated by $C_{0}$. Since $C_{0}$ is separable, $C_{1}$ has a strictly positive element. Since $A$ and $B$ are full hereditary subalgebras of $C_{1}, 2.8$ implies $A$ and $B$ are stably isomorphic.

CoRollary 2.10. If $A$ is a separable $C^{*}$-algebra and $i: B \rightarrow A$ is the inclusion of a full hereditary $C^{*}$-subalgebra, then $i^{*}: \operatorname{Ext}(A) \rightarrow$ $\operatorname{Ext}(B)$ is an isomorphism.

Proof. Let $C$ be the $C^{*}$-algebra constructed in the proof of 2.8, and define $j_{1}: B \rightarrow C$ and $j_{2}: A \rightarrow C$ by $j_{1}(b)=b \otimes e_{11}$ and $j_{2}(a)=a \otimes e_{22}$. By 2.7 $j_{1}^{*}$ and $j_{2}^{*}$ are isomorphisms. Thus it is sufficient to prove $\left(j_{2} \circ i\right)^{*}$ is an isomorphism, and for this it is sufficient to show $\left(j_{2} \circ i\right)^{*}=j_{1}^{*}$. But $j_{1}$ and $j_{2} \circ i$ both have images contained in $B \otimes M_{2} \subset C$. If $k_{1}$ and $k_{2}$ are the corresponding maps from $B$ to $B \otimes M_{2}$, it is sufficient to show $k_{1}^{*}=k_{2}^{*}$, and this follows directly from $[6,3.11]$.

It is interesting to look for invariants under stable isomorphism. Among the obvious ones are separability, existence of a strictly positive element, existence of a faithful state, the spectrum, Ext, being of continuous trace, the element of $\breve{H}^{3}(\hat{A}, Z)$ corresponding to a continuous trace algebra $A$, and being of generalized continuous trace $([10,4.7 .12])$. One noninvariant property that comes immediately to mind is the existence of (enough) finite-diminsional representations. This suggests that one attempts to determine which $C^{*}$ algebras are stably isomorphic to algebras with finite-dimensional representations. For the remainder of this section we assume for simplicity that all $C^{*}$ - algebras considered are separable.

Let $F D$ be the class of $C^{*}$-algebras all of whose irreducible representations are finite dimensional, $B D_{n}$ the class of whose irreducible representations have dimension $\leqq n$, and $B D=\bigcup_{n=1}^{\infty} B D_{n}$. The basic method for using our theorem to prove that $A$ is stably isomorphic to an algebra in $F D$, for example, is to find an $e \in A$ such that $\pi(e)$ is nonzero and of finite rank for each irreducible representation $\pi$. Then $B=\overline{e A e}$ fulfils the hypotheses of 2.8 and $B \in F D$. The next corollary contains some examples of what can be proved with this technique, though it is not best possible. 
CoRollary 2.11.3,4 Let $A$ be a separable CCR $C^{*}$-algebra.

(a) If $\hat{A}$ is either Hausdorff or (quasi-) compact, then $A$ is stably isomorphic to an algebra in FD.

(b) In particular if $A$ has continuous trace, (a) applies.

(c) If $A$ has continuous trace and $\hat{A}$ is compact, then $A$ is stably isomorphic to an algebra in $B D$.

(d) If $A$ has continuous trace and $\hat{A}$ has finite topological dimension $n$, then $A$ is stably isomorphic to an algebra in $B D_{n+1}$.

(e) If $A$ has continuous trace and $\hat{A}$ is homeomorphic to $S^{n}$, then $A$ is stably isomorphic to an algebra in $B D_{2}$.

\section{Sketch of proof.}

(a) For $\pi_{0} \in \hat{A}$, choose $e_{0} \geqq 0$ such that $\pi_{0}\left(e_{0}\right) \neq 0$. Let $g$ be a nonnegative continuous function on $\boldsymbol{R}$ such that $g\left(\left\|\pi_{0}\left(e_{0}\right)\right\|\right) \neq 0$ and $g$ vanishes on a neighborhood of 0 , and let $f_{0}=g\left(e_{0}\right)$. Then $\pi_{0}\left(f_{0}\right) \neq 0$ and rank $\pi\left(f_{0}\right)$ is finite for all $\pi \in \hat{A}$. The sets $\left\{\pi \in \hat{A}: \pi\left(f_{0}\right) \neq 0\right\}$ form an open cover of $\hat{A}$. We now use either a compactness or paracompactness argument.

(c) The element $f_{0}$ above can be chosen so that rank $\pi\left(f_{0}\right) \leqq 1$ for all $\pi$.

(d) Every open cover of $\hat{A}$ has a refinement such that no point of $\hat{A}$ is in more than $n+1$ sets of the refinement.

(e) $S^{n}$ can be covered by two open sets such that $A$ is trivial over each. (Note that if $A$ is stable and of continuous trace then according to the results of [12] $A$ arises from a locally trivial $\mathscr{K}$ bundle.)

\section{REMARKS.}

1. The purpose of $2.11(\mathrm{e})$ (which is trivial for $n \neq 3$ ) is to improve the counterexample in 6.28 of [5]. The algebra there could be taken in $B D_{2}$.

2. It follows from [11] that any algebra in $F D$ is of generalized continuous trace. P. Green has recently proved, by combining the technique of 2.11 with a result on the topology of the spectrum of a generalized continuous trace algebra, that every (separable) generalized continuous trace algebra is stably isomorphic to an element of $F D$.

3. It is not hard to prove, using [12] and a result of Serre that a continuous trace algebra $A$, is stably isomorphic to a $C^{*}$ algebra with unit if and only if $\hat{A}$ is compact and the corresponding

${ }^{3}$ We are grateful to P. Green for suggesting the elimination of a redundant hypothesis from (a).

4 There is some overlap between points (c), (d), and (e) and results of M. Dupré, Cohomology for classifying categories of Banach bundles, Summary, $\$ 6$ (preprint). 
element of $\check{H}^{3}(\hat{A}, \boldsymbol{Z})$ is torsion. The same problem for general $C^{*}$ algebras appear to be difficult.

3. Relations with other subjects. This paper was motivated by $[6, \S 3]$. The stable isomorphisms found there between a separable $C^{*}$-algebra $A$ and a subalgebra $B$ can be put into the following abstract context: $A=C \otimes \mathscr{K}$ and $B$ is the full corner defined by a projection $p \in M(C \otimes \mathscr{K})$ such that $p$ commutes with $C \otimes 1$. In this case $p$ arises from a continuous function from $\hat{C}$ to $\mathscr{G}$ and hence defines a continuous field of Hilbert spaces, $E$, over $\hat{C}$. [12, Cor. 3, p. 260] implies that $E \oplus E \oplus \cdots$ is trivial, which implies the stable isomorphism. It is now clear that the hypothesis that $p$ commute with $C \otimes 1$ is unnecessary for the stable isomorphism (though it is necessary for the purposes of $[6, \S 4]$ ). On the other hand, the application of the result of [12] gives a stronger conclusion than our results in this context. The extra strength is in the fact that the partial isometry $v$ of 2.5 also commutes with $C \otimes 1 \subset$ $M(A \otimes \mathscr{K})$. Note, though, that this would be automatic if $C$ is commutative, since then $C \otimes 1 \otimes 1$ is central in $M(C \otimes \mathscr{K} \otimes \mathscr{K})$. The upshot of this is that there is significant overlap between our results and a portion of [12] but neither implies the other.

In general, there is a fairly good analogy between our results and [12]. In order to elaborate this, we state explicitly two results of [12]:

I. If $E$ is a separable continuous field over $X$ and $E_{0}$ is a trivial field of countably infinite rank over $X$, where $X$ is paracompact, then $E \oplus E_{0} \cong E_{0}$ (Thm. 4, p. 259).

II. If $E$ is separable, $E_{x} \neq 0$ for each $x \in X$, and $X$ is paracompact, then $E \oplus E \oplus \cdots$ is trivial (Cor. 3, p. 260).

The hypotheses that $E$ be separable and $X$ paracompact are analogous to existence of a strictly positive element. The hypothesis that $E_{x} \neq 0$ for each $x$ is analogous to fullness. A corner is analogous to a complemented subfield, and a hereditary $C^{*}$-subalgebra is analogous to an arbitrary subfield. (If $X$ is locally compact Hausdorff this is more than just an analogy.) Thus our main theorem is analogous to II. It may seem that we are dealing only with the special case of II where $E$ is known a priori to be a subfield of a trivial field, but actually this is no restriction because of the level of abstractness. If $E$ and $E^{\prime}$ are two fields over $X$ such that $E_{x} \neq 0$ and $E_{x}^{\prime} \neq 0$ for all $x \in X$, then we may form $E \oplus E^{\prime}$. If $A, A^{\prime}$, and $C$ are the corresponding $C^{*}$-algebras (change the word "corresponding" to "analogous" if $X$ is not locally compact Hausdorff), then $A$ and $A^{\prime}$ are 
full corners of $C$ and the relationship is the same as that between $A$, $B$, and $C$ in the proof of 2.8 (except that there is no inclusion relation between $A$ and $A^{\prime}$ ). Thus 2.8 and indeed 2.6 is an adequate analogue of II. We also see that given two $C^{*}$-algebras $A$ and $A^{\prime}$, the formation of a $C^{*}$-algebra $C$ like that of 2.8 is analogous to the formation of the direct sum of two fields. The existence of such a $C$ is analogous to the fact of two fields having homeomorphic base spaces. $C$ may not be unique. (Note that one needs to choose a specific homeomorphism between the base spaces before one can form the direct sum of fields with different base spaces.)

It seems appropriate to ask, "Under what conditions is it possible to construct a $C^{*}$-algebra $C$ having two given $C^{*}$-algebras $A$ and $A^{\prime}$ as "opposite" full corners?" One answer to this question is provided by the concept of Morita equivalence. See [7] for details.

Thus far our analogy has not mentioned I. Although I was used in the proof of II in [12], we note that I follows from II in the special case where $E_{x} \neq 0$ for all $x$. To formulate an analogue of $\mathrm{I}$ in general, we assume $B$ is a hereditary $C^{*}$-subalgebra of $A$ and define a $C^{*}$-algebra $D \subset A \otimes \mathscr{K}$ as follows: If $d \in A \otimes \mathscr{K}$ and $d_{i j} \otimes e_{i j}=\left(1 \otimes e_{i i}\right) d\left(1 \otimes e_{j j}\right)$, then $d \in D$ if and only if $d_{11} \in B, d_{1 j} \in \overline{B \cdot A}$, and $d_{i 1} \in \overline{A \cdot B}$. $\quad B$ is a corner of $D$ defined by the projection $p$ which may be symbolized by $1 \otimes e_{11}$ (where " 1 " is the identity of $M(B)$ ). $A \otimes \mathscr{K}$ can be identified with the opposite corner, which is defined by $1-p=\sum_{2}^{\infty} 1 \otimes e_{j j}$ (where " 1 " is the identity of $M(A)$ ).

THEOREM 3.1. If $B$ has a strictly positive element, then there is a partial isometry $u \in M(D)$ such that $u^{*} u=1-p$ and $u u^{*}=1$.

Sketch of proof. Let $e$ be a strictly positive element of $B$. Then $u$ can be defined by the infinite matrix:

$$
\left(\begin{array}{cccccc}
0 & e & \left(1-e^{2}\right)^{1 / 2} e & \left(1-e^{2}\right) e & \left(1-e^{2}\right)^{3 / 2} e & \cdots \\
0 & -\left(1-e^{2}\right)^{1 / 2} & e^{2} & \left(1-e^{2}\right)^{1 / 2} e^{2} & \left(1-e^{2}\right) e^{2} & \cdots \\
0 & 0 & -\left(1-e^{2}\right)^{1 / 2} & e^{2} & \left(1-e^{2}\right)^{1 / 2} e^{2} & \cdots \\
0 & 0 & 0 & -\left(1-e^{2}\right)^{1 / 2} & e^{2} & \cdots \\
0 & 0 & 0 & 0 & -\left(1-e^{2}\right)^{1 / 2} & \cdots \\
\cdot & . & . & . & . & \cdots \\
. & . & . & . & . & \cdots \\
\cdot & . & . & . & . & \cdots \\
. & . & . & . & . & \cdots
\end{array}\right)
$$

Before proceeding further, we prove some things needed to justify some of the above remarks. Let $A$ and $B$ be $C^{*}$-algebras, and let $\lambda: B \rightarrow \mathscr{L}(\mathscr{H})$ be a faithful representation. If $\pi$ is any 
irreducible representation of $A$, then $\pi \otimes \lambda$ is a representation of $A \otimes_{\min } B$. The direct sum of $\pi \otimes \lambda$ for all $\pi \in \hat{A}$ is faithful, since it is $\left(\sum_{\pi \in \hat{A}}^{\oplus} \pi\right) \otimes \lambda$. Thus if $f: \hat{A} \rightarrow B$, and $a \in A$, there can be at most one $c \in A \otimes_{\min } B$ such that $(\pi \otimes \lambda)(c)=(\pi \otimes \lambda)(a \otimes f(\pi))$ for all $\pi \in \hat{A}$. This $c$ (if it exists) will be devoted $a \otimes f$. It is easily seen that the existence and identity of $a \otimes f$ is independent of the choice of $\lambda$.

Lemma 3.2. $a \otimes f$ exists for all $a \in A$ if and only if $f$ is bounded and continuous.

Proof. Assume $a \otimes f$ exists for all $a$. The closed graph theorem implies that the linear map $a \rightarrow a \otimes f$ is bounded and hence that $f$ is bounded. To any state $\psi$ on $A$ corresponds a linear contraction $R_{\psi}: A \otimes_{\min } B \rightarrow B$ such that $R_{\psi}(a \otimes b)=\psi(a) \cdot b$ [16]. If $\pi$ is a representation of $A$ and $\theta$ a cyclic vector such that $\psi(\alpha)=(\pi(\alpha) \theta, \theta)$ for all $a \in A$, then $R_{\psi}$ can be constructed by identifying $\lambda \circ R_{\psi}$ with the compression of $\pi \otimes \lambda$ to $\boldsymbol{C} \theta \otimes \mathscr{H}$. If $\psi$ is a pure state, $R_{\psi}(a \otimes f)=\psi(a) f(\pi)$. Also the assignment $\psi \rightarrow R_{\psi}$ is continuous from the weak* topology to the strong operator topology. Now if a net $\left\{\pi_{\alpha}\right\}$ converges to $\pi$ in $\hat{A}$, choose a state $\psi$ defining $\pi$ and an $a \in A$ such that $\psi(a) \neq 0$. Then by passing to a subnet if necessary, we can choose states $\psi_{\alpha}$ defining $\pi_{\alpha}$ such that $\psi_{\alpha} \rightarrow \psi$ weak ${ }^{*}$ ([10, 3.4.11]). Thus $R_{\psi_{\alpha}}(a \otimes f)=\psi_{\alpha}(a) f\left(\pi_{\alpha}\right) \rightarrow \psi(a) f(\pi)=R_{\psi}(a \otimes f)$, and hence $f\left(\pi_{\alpha}\right) \rightarrow f(\pi)$. Therefore $f$ is continuous.

Now assume $f$ is bounded and continuous, $a \in A$, and further that $\|a\|=\|f\|_{\infty}=1$. For each $n=1,2, \cdots$ let $K_{n}=\{\pi \in \hat{A}$ : $\|\pi(a)\| \geqq 1 / n\}$ and let $L_{n}$ be the closed convex hull of $f\left(K_{n}\right) . \quad L_{n}$ is compact since $K_{n}$ is. By the Dugundji extension theorem ([13]) there is a continuous retraction $r_{n}: B \rightarrow L_{n}$. Let $\left\{U_{i}\right\}$ be a finite open cover of $L_{n}$ consisting of sets of diameter less than $1 / n$, and let $\left\{\phi_{i}\right\}$ be a partition of unity for $L_{n}$ subordinate to $\left\{U_{i}\right\}$. Choose $b_{i} \in L_{n} \cap U_{i}$ for each $i$ and define $g_{n}: \hat{A} \rightarrow B$ by $g_{n}(\pi)=\sum\left(\phi_{i} \circ r_{n} \circ f(\pi)\right) \cdot b_{i}$. Then $a \otimes g_{n}$ exists, since it is $\sum\left(\phi_{i} \circ r_{n} \circ f\right) a \otimes b_{i}$. Here we are using the Dauns-Hofmann theorem (see [14] and references), which implies that for any bounded continuous scalar valued function $h$ on $\hat{A}$ there is an element $h \alpha$ of $A$ such that $\pi(h \alpha)=h(\pi) \pi(\alpha)$ for all $\pi \in \hat{A}$. In this case $h=\phi_{i} \circ r_{n} \circ f$. If $\pi \in K_{n}$, then $\left\|g_{n}(\pi)-f(\pi)\right\|<1 / n$. Thus for all $\pi \in \hat{A}\left\|(\pi \otimes \lambda)\left(\alpha \otimes g_{n}\right)-(\pi \otimes \lambda)(\alpha \otimes f(\pi))\right\|<2 / n$. It follows that $\left\{a \otimes g_{n}\right\}$ is a Cauchy sequence and its limit fulfils the definition of $a \otimes f$.

THEOREM 3.3. The elements of $M\left(A \bigotimes_{\min } B\right)$ which commute with $A \otimes 1$ can be identified naturally with the bounded strictly continuous functions $F: \hat{A} \rightarrow M(B)$. 
Proof. If $x \in M\left(A \otimes_{\min } B\right)$ commutes with $A \otimes 1$, then for each $\pi \in \hat{A}(\pi \otimes \lambda)^{\sim}(x)$ commutes with $\pi(A) \otimes 1$. Thus $(\pi \otimes \lambda)^{\sim}(x)=1 \otimes$ $T(\pi), \quad$ for some $T(\pi) \in \mathscr{L}(\mathscr{H}) . \quad(1 \otimes T(\pi)) \cdot(\pi(a) \otimes \lambda(b))=\pi(a) \otimes$ $T(\pi) \lambda(b)$ and $(\pi(a) \otimes \lambda(b))(1 \otimes T(\pi))=\pi(a) \otimes \lambda(b) T(\pi)$ are each in $(\pi \otimes \lambda)\left(A \otimes_{\min } B\right)$ for all $a \in A, b \in B$. It follows that $T(\pi) \lambda(b)$ and $\lambda(b) T(\pi)$ are in $\lambda(B)$. (Choose a unit vector $\theta$ such that $\psi(a)=$ $(\pi(a) \theta, \theta) \neq 0$ and apply $R_{\psi}$.) This means that $T(\pi)=\tilde{\lambda}(F(\pi))$ for some unique $F(\pi) \in M(B)$. (See [3, p. 277] for example.) Now for each $b \in B$ we have functions $F b$ and $b F$ from $\hat{A}$ to $B$ defined by $\pi \rightarrow F(\pi) b$ and $\pi \rightarrow b F(\pi)$. We have just seen that $x(a \otimes b)$ fulfils the definition of $a \otimes F b$ and $(a \otimes b) x$ fulfils the definition of $a \otimes b F$. Thus 3.2 implies $F b$ and $b F$ are continuous, and hence $F$ is strictly continuous. It is clear that $x$ is determined by $F$.

Conversely, suppose $F$ (bounded, strictly continuous) is given. We wish to define bounded linear maps $L, R: A \bigotimes_{\min } B \rightarrow A \bigotimes_{\min } B$ such that $L(a \otimes b)=a \otimes F b$ and $R(a \otimes b)=a \otimes b F$ for all $a \in A$, $b \in B$. Since these expressions are defined by 3.2 and bilinear on $A \times B, L$ and $R$ are defined on the algebraic tensor product. Since $\|c\|=\sup \{\|(\pi \otimes \lambda)(c)\|: \pi \in \hat{A}\}$, we see that $\|L\|,\|R\| \leqq\|F\|_{\infty}$. Hence $L$ and $R$ can be defined on $A \otimes_{\min } B$. Again applying $\pi \otimes \lambda$, we see easily that $c L\left(c^{\prime}\right)=R(c) \cdot c^{\prime}$, so that the pair $(L, R)$ defines an element $x \in M\left(A \otimes_{\min } B\right)$. It is obvious that

$$
(\pi \otimes \lambda)^{\sim}(x)=(\pi \otimes \lambda)^{\sim}(1 \otimes F(\pi)),
$$

so that $x$ commutes with $A \otimes 1$ and the proof is complete.

It is known that the topological product $\hat{A} \times \hat{B}$ is homeomorphically identified with a dense subset of $\left(A \boldsymbol{\otimes}_{\min } B\right)^{\wedge}$. A byproduct of 3.3 is that these spaces have the same Stone-Čech compactification. ${ }^{5}$

CoROLlaRY 3.4. Every bounded continuous complex-valued function on $\hat{A} \times \hat{B}$ extends to $\left(A \otimes_{\min } B\right)^{\wedge}$.

Proof. We compute the center, $Z$, of $M\left(A \otimes_{\min } B\right) . \quad x \in Z$ if and only if $x$ commutes with $A \otimes 1$ and $1 \otimes B$. Thus $x$ is given by $F: \hat{A} \rightarrow M(B)$, and the range of $F$ must be in the center of $M(B)$. Since the Dauns-Hofmann theorem identifies the center of $M(B)$ with $C_{b}(\hat{B})$, the space of bounded continuous complex-valued functions on $\hat{B}, x$ is described by a bounded strictly continuous function from $\hat{A}$ to $C_{b}(\hat{B})$. But a direct application of the Dauns-Hofmann theorem identifies $Z$ with $C_{b}\left(A \otimes_{\min } B\right)^{\wedge}$. Thus we need only show that the bounded strictly continuous functions from $\hat{A}$ to $C_{b}(\hat{B})$ are the same as the elements of $C_{b}(\hat{A} \times \hat{B})$. We need:

${ }^{5}$ In [4] it is shown that if $A$ or $B$ is nuclear, then $\operatorname{Prim}(A \otimes B)=\operatorname{Prim} A \times \operatorname{Prim} B$. 
LEMMA 3.5. The restriction of the strict topology of $M(B)$ to a bounded subset of $C_{b}(\hat{B})$ coincides with the topology of uniform convergence on compact sets.

Proof. Suppose $\left\{f_{\alpha}\right\}$ is a net in $C_{b}(\hat{B}), f_{\alpha} \rightarrow f$ uniformly on compact subsets, $\left\|f_{\alpha}\right\|_{\infty} \leqq M$ for all $\alpha$, and $b \in B$. For $\varepsilon>0$ let $K_{\varepsilon}=$ $\{\rho \in \hat{B}:\|\rho(b)\| \geqq \varepsilon\}$. Then $K_{\varepsilon}$ is compact. $\left\|\left(f_{\alpha}-f\right) b\right\|=\sup \left\{\mid f_{\alpha}(\rho)-\right.$ $f(\rho) \mid\|\rho(b)\|: \rho \in \widehat{B}\} \leqq \max \left(\sup \left\{\left|f_{\alpha}(\rho)-f(\rho)\right|: \rho \in K_{\varepsilon}\right) \cdot\|b\|, 2 \in M\right)$. Since $\varepsilon$ is arbitrary, this implies $f_{\alpha} b \rightarrow f b$. Hence $f_{\alpha} \rightarrow f$ strictly.

Conversely, assume $f_{\alpha} \rightarrow f$ strictly. For any compact $K$ there exists $0 \leqq e \in B$ such that $\|\rho(e)\| \geqq 1$ for $\rho \in K$. (This is an easy argument using the lower semi-continuity of $\|\rho(e)\|$.$) Then f_{\alpha} e \rightarrow f e$ clearly implies that $f_{\alpha} \rightarrow f$ uniformly on $K$.

Now an elementary point-set topological argument completes the proof of 3.4. We need the local (quasi-) compactness of $\hat{B}$, but the fact that $\hat{B}$ may not be Hausdorff causes no difficulty.

We now return to the case where $A=C \otimes \mathscr{K}$ and $p$ is a projection in $M(A)$ which commutes with $C \otimes 1$. In [6, §4] similar hypotheses were used to construct an endomorphism $m_{p}$ of $\operatorname{Ext}(C) . m_{p}$ is constructed by following the natural map $\operatorname{Ext}(C) \cong \operatorname{Ext}(C \otimes \mathscr{K})$ with the map $\zeta^{*}: \operatorname{Ext}(A) \rightarrow \operatorname{Ext}(C)$, where $\zeta: C \rightarrow A$ is defined by $\zeta(c)=(c \otimes 1) \cdot p$. In order for this to make sense, we need $(c \otimes 1) p$ to be in $A$ for all $c \in C$. According to 3.2 and the proof of 3.3 this happens precisely when the map $P: \hat{C} \rightarrow \mathscr{G}$ which defines $p$ satisfies:

1. $P(\pi)$ is finite rank for all $\pi \in \hat{C}$.

2. $P$ is norm-continuous. $m_{p_{1}}$ will equal $m_{p_{2}}$ if there is a partial isometry $u \in M(A)$ such that $u$ commutes with $C \otimes 1, u^{*} u=p_{1}$ and $u u^{*}=p_{2}$. Thus we are dealing with a class of ordinary vector bundles over $\hat{C}$. The bundles which arise are precisely the pull-backs of bundles over the complete regularization of $\hat{C}$. Note that none of the results of $\S 2$ are needed to define $m_{p}$.

\section{REFERENCES}

1. J. Aarnes and R. V. Kadison, Pure states and approximate identities, Proc. Amer. Math. Soc., 21 (1969), 749-752.

2. C. A. Akemann, Left ideal structure of $C^{*}$-algebras, J. Functional Analysis, 6 (1970), 305-317.

3. - G. K. Pedersen, and J. Tomiyama, Multipliers of $C^{*}$-algebras, J. Functional Analysis, 13 (1973), 277-301.

4. B. Blackadar, Infinite tensor products of $C^{*}$-algebras, preprint.

5. L. G. Brown, Extensions and the structure of $C^{*}$-algebras, Symp. Mat., 20 (1976), 539-566.

6. - R. G. Douglas and P. A. Fillmore, Extensions of $C^{*}$-algebras and $K$ homology, Ann. Math., 105 (1977), 265-324.

7. - P. Green and M. A. Rieffel, Stable isomorphism and strong Morita equiva- 
lence of $C^{*}$-algebras, Pacific J. Math., 71 (1977), 349-363.

8. R. C. Busby, Double centralizers and extensions of $C^{*}$-algebras, Trans. Amer. Math. Soc., 132 (1968), 79-99.

9. M. D. Choi and E. A. Effros, The completely positive lifting problem for $C^{*}$-algebras, Ann. Math., 104 (1976), 585-609.

10. J. Dixmier, Les $C^{*}$-algébres et Leurs Représentations, Gauthier-Villars, Paris, 1964. 11. - Points séparés dans le spectre d'une $C^{*}$-algébre, Acta Sci. Math., (Szeged) 22 (1961), 115-128.

12. J. Dixmier and A. Douady, Champs continus d'espaces hilbertiens et de C*-algébres, Bull. Soc. Math. France, 91 (1963), 227-284.

13. J. Dugundji, An extension of Tietze's theorem, Pacific J. Math., 1 (1951), 353-367. 14. G. A. Elliott and D. Olesen, A simple proof of the Dauns-Hofmann theorem, Math. Scand., 34 (1974), 231-234.

15. C. Lance, On nuclear $C^{*}$-algebras, J. Functional Analysis, 12 (1973), 157-176.

16. J. Tomiyama, Applications of a Fubini type theorem to the tensor products of $C^{*}$ algebras, Tôhoku Math. J., 19 (1967), 213-226.

17. D. Voiculescu, A non-commutative Weyl-von Neumann theorem, Rev. Roum. Math., 21 (1976), 97-113.

Received November 22, 1976. This paper was written while the author was visiting at the University of California at Berkeley and was partially supported by grants from the Alfred P. Sloan Foundation and National Science Foundation.

Purdue University

WEST LAFAYETTE, IN 47907 


\section{PACIFIC JOURNAL OF MATHEMATICS}

\section{EDITORS}

RICHARD ARENS (Managing Editor)

University of California

Los Angeles, CA 90024

Charles W. Curtis

University of Oregon

Eugene, OR 97403

C. C. MOORE

University of California

Berkeley, CA 94720

\section{J. DugundJI}

Department of Mathematics

University of Southern California

Los Angeles, CA 90007

R. FinN and J. Milgram

Stanford University

Stanford, CA 94305

ASSOCIATE EDITORS
E. F. BECKENBACH
B. H. NeumanN
F. WOLF
K. YOSHIDA

\section{SUPPORTING INSTITUTIONS}

UNIVERSITY OF BRITISH COLUMBIA CALIFORNIA INSTITUTE OF TECHNOLOGY

UNIVERSITY OF CALIFORNIA

MONTANA STATE UNIVERSITY

UNIVERSITY OF NEVADA, RENO

NEW MEXICO STATE UNIVERSITY

OREGON STATE UNIVERSITY

UNIVERSITY OF OREGON

OSAKA UNIVERSITY

\author{
UNIVERSITY OF SOUTHERN CALIFORNIA \\ STANFORD UNIVERSITY \\ UNIVERSITY OF HAWAII \\ UNIVERSITY OF TOKYO \\ UNIVERSITY OF UTAH \\ WASHINGTON STATE UNIVERSITY \\ UNIVERSITY OF WASHINGTON \\ AMERICAN MATHEMATICAL SOCIETY
}

The Supporting Institutions listed above contribute to the cost of publication of this Journal, but they are not owners or publishers and have no responsibility for its content or policies.

Mathematical papers intended for publication in the Pacific Jaurnal of Mathematics should be in typed form or offset-reproduced, (not dittoed), double spaced with large margins. Please do not use built up fractions in the text of your manuscript. You may however, use them in the displayed equations. Underline Greek letters in red, German in green, and script in blue. The first paragraph or two must be capable of being used separately as a synopsis of the entire paper. Items of the bibliography should not be cited there unless absolutely necessary, in which case they must be identified by author and Journal, rather than by item number. Manuscripts, in triplicate, may be sent to any one of the editors. Please classify according to the scheme of Math. Reviews, Index to Vol. 39. All other communications should be addressed to the managing editor, or Elaine Barth, University of California, Los Angeles, California, 90024.

The Pacific Journal of Mathematics expects the author's institution to pay page charges, and reserves the right to delay publication for nonpayment of charges in case of financial emergency.

100 reprints are provided free for each article, only if page charges have been substantially paid. Additional copies may be obtained at cost in multiples of 50 .

The Pacific Journal of Mathematics is issued monthly as of January 1966. Regular subscription rate: $\$ 7200$ a year (6 Vols., 12 issues). Special rate: $\$ 36.00$ a year to individual members of supporting institutions.

Subscriptions, orders for back numbers, and changes of address should be sent to Pacific Journal of Mathematics, 103 Highland Boulevard, Berkeley, California, 94708.

PUBLISHED BY PACIFIC JOURNAL OF MATHEMATICS, A NON-PROFIT CORPORATION

Printed at Kokusai Bunken Insatsusha (International Academic Printing Co., Ltd.). 8-8, 3-chome, Takadanobaba, Shinjuku-ku, Tokyo 160, Japan.

Copyright (C) 1975 by Pacific Journal of Mathematics Manufactured and first issued in Japan 


\section{Pacific Journal of Mathematics}

\section{Vol. 71, No. $2 \quad$ December, 1977}

Krishnaswami Alladi and Paul Erdős, On an additive arithmetic

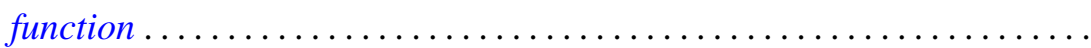

James Bailey and Dale Rolfsen, An unexpected surgery construction of a

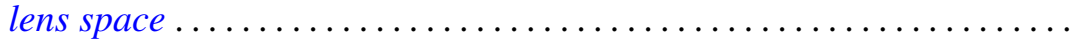

Lawrence James Brenton, On the Riemann-Roch equation for singular

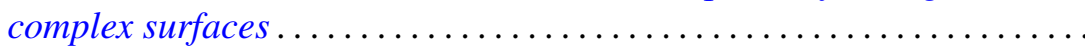

James Glenn Brookshear, Projective ideals in rings of continuous

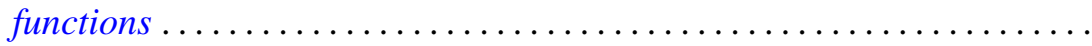

Lawrence Gerald Brown, Stable isomorphism of hereditary subalgebras of

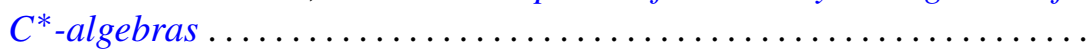

Lawrence Gerald Brown, Philip Palmer Green and Marc Aristide Rieffel, Stable isomorphism and strong Morita equivalence of $C^{*}$-algebras....

N. Burgoyne, Robert L. Griess, Jr. and Richard Lyons, Maximal subgroups

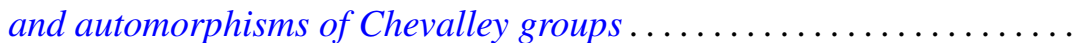
365

Yuen-Kwok Chan, Constructive foundations of potential theory .... 405

Peter Fletcher and William Lindgren, On $w \Delta$-spaces, $w \sigma$-spaces and

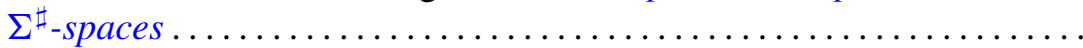

Louis M. Friedler and Dix Hayes Pettey, Inverse limits and mappings of

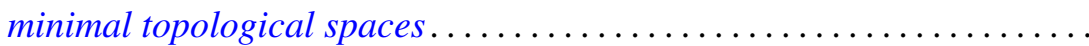

Robert E. Hartwig and Jiang Luh, A note on the group structure of unit regular ring elements.

I. Martin (Irving) Isaacs, Real representations of groups with a single involution ...

Nicolas P. Jewell, The existence of discontinuous module derivations . .

Antonio M. Lopez, The maximal right quotient semigroup of a strong semilattice of semigroups .......................

Dennis McGavran, $T^{n}$-actions on simply connected $(n+2)$-manifolds

Charles Anthony Micchelli and Allan Pinkus, Total positivity and the exact $n$-width of certain sets in $L^{1}$.

Barada K. Ray and Billy E. Rhoades, Fixed point-theorems for mappings with a contractive iterate .......................

Fred Richman and Elbert A. Walker, Ext in pre-Abelian categories. .

Raymond Craig Roan, Weak* generators of $H^{\infty}$ and $l^{1}$..

Saburou Saitoh, The exact Bergman kernel and the kernels of Szegö type...

Kung-Wei Yang, Operators invertible modulo the weakly compact 